\title{
Greater preferences for death in hospital and mechanical ventilation at the end of life among non-whites recently diagnosed with cancer
}

\author{
David Boyce-Fappiano' ${ }^{1}$ Kaiping Liao ${ }^{2} \cdot$ Christopher Miller $^{3} \cdot$ Susan K. Peterson ${ }^{3} \cdot$ Linda S. Elting $^{2}$. \\ B. Ashleigh Guadagnolo ${ }^{1,2} \mathbb{D}$
}

Received: 22 January 2021 / Accepted: 13 April 2021 / Published online: 29 April 2021

(c) The Author(s), under exclusive licence to Springer-Verlag GmbH Germany, part of Springer Nature 2021

\begin{abstract}
Purpose Non-white cancer patients receive more aggressive care at the end-of-life (EOL). This may indicate low quality EOL care if discordant with patient preferences. We investigated preferred potential place of death and preferences regarding use of mechanical ventilation in a cohort of Texas cancer patients.

Methods A population-based convenience sample of recently diagnosed cancer patients from the Texas Cancer Registry was surveyed using a multi-scale inventory between March 2018 and June 2020. Item responses to questions about preferences regarding location of death and mechanical ventilation were the outcome measures of this investigation. Inverse probability weighting analysis was used to construct multivariable logistic regression examining the associations of covariates.

Results Of the 1460 respondents, a majority ( $82 \%$ ) preferred to die at home compared to $8 \%$ who preferred dying at the hospital. In total, $25 \%$ of respondents expressed a preference for undergoing mechanical ventilation at the EOL. Adjusted analysis showed increased preference among Black (OR $=1.81$; 95\% CI: 1.19-2.73) and other non-white, non-Hispanic race individuals (OR $=3.53$; 95\% CI: 1.99-6.27) for dying at a hospital. Males, married individuals, those of higher education and poor self-reported health showed significantly higher preference for dying at home. Non-white respondents of all races were more likely to prefer mechanical ventilation at the EOL as were individuals who lived with another person at home.

Conclusion Non-white cancer patients were more likely to express preferences coinciding with aggressive EOL care including dying at the hospital and utilizing mechanical ventilation. These findings were independent of other sociodemographic characteristics, including decisional self-efficacy.
\end{abstract}

Keywords Cancer $\cdot$ Mechanical ventilation $\cdot$ End of life $\cdot$ Place of death

\section{Introduction}

Cancer patients are more likely to experience aggressive near-death treatment including receiving EOL chemotherapy, having emergency department visits, and intensive care unit (ICU) admissions with mechanical ventilation [1, 2].

B. Ashleigh Guadagnolo aguadagn@mdanderson.org

1 Department of Radiation Oncology, MD Anderson Cancer Center, 1515 Holcombe Blvd. Unit 97, Houston, TX 77030, USA

2 Department of Health Services Research, MD Anderson Cancer Center, 1515 Holcombe Blvd. Unit 97, Houston, TX 77030, USA

3 Department of Behavioral Science, MD Anderson Cancer Center, Houston, TX, USA
Previous studies have shown that non-white cancer patients disparately receive more aggressive end-of-life (EOL) care defined as admission to higher acuity care in their final days, lack of hospice utilization, and increased use of mechanical ventilation [3-5]. Not only are these aggressive measures indicative of potentially lower quality EOL care [1, 6], but they also raise the question of whether racial and ethnic minorities are experiencing what the Institute of Medicine (IOM) terms "a good death" [7]. Furthermore, overly aggressive end-of-life (EOL) measures can result in elevated health care costs, negatively impact patients' quality of life and have detrimental effects on patients loved ones $[4,8-10]$. This issue is more apparent in cancer patients who often require early and more expansive EOL care due to the often predictable and chronic decline inherent in the disease process for those who are not cured [11, 12]. 
Findings that non-white cancer patients receive more aggressive care at the EOL raise the question of whether lack of access to effective palliative care and hospice programs contribute to this disparity [3]. However, a recent study of Texas cancer patients examined racial differences in hospice utilization and displayed that despite accounting for the use of fee-for-service and Medicare payment, racial disparities in hospice enrollment persisted [5]. This raised the question of whether preferences for more aggressive care were contributing to observed EOL care disparities. Evidence suggests that cancer patients who participate in advance care planning or effective EOL discussions with their providers will aim for quality and comfort-based care with the goals of avoiding aggressive measures such as mechanical ventilation and death within the ICU. [8, 13, 14] However, programs and policy initiatives may need to dedicate efforts toward culturally responsive educational and social support for non-white patients who may arrive at terminal illness with preferences for more aggressive or potentially futile care [15].

In order to better understand EOL care variation among a racially, geographically, and socio-economically diverse population, we conducted a registry-based survey study and examined cancer patient preferences regarding dying at home versus in the hospital as well as use of mechanical ventilation in a large cohort of recently diagnosed Texas cancer patients.

\section{Methods}

\section{Data source, study sample and data collection procedure}

This survey study is part (Project 4) of the larger multi-year Comparative Effectiveness Research on Cancer in Texas (CERCIT) program, funded by the Cancer Prevention and Research Institute of Texas (CPRIT) (https://www.utmb. edu/scoa/research/supported-research-programs/compa rative-effectiveness-research-on-cancer-in-texas/currentprojects).[16] We recruited potential survey respondents from the state-wide population-based Texas Cancer Registry (TCR) ((https://www.dshs.state.tx.us/tcr/).[17] A sample of 6222 TCR registrants 18 years of age and older with a diagnosis of a solid tumor malignancy in the past 12 months were obtained. All stages of cancer were included. Of these, 5535 were determined to be alive and able to receive study questionnaires by mail delivery. In total, 1566 individuals responded between March 2018 and July 2020, yielding a response rate of $28.3 \%$. Of these respondents, 1460 (93.2\%) were included for this analysis because they provided complete responses to the survey items for the study. We performed telephone follow-up early in the data collection time period in an attempt to increase/solicit further participation, however yield was very low $(<10 \%)$. For the first 2300 potential participants, we sent three follow-up reminders and two replacement study questionnaires at 2 weeks, 4-6 weeks, and 8-10 weeks to non-respondents. During the course of this study's data collection efforts, the TCR/Texas Department of State Health Services changed their policy regarding the number of allowable attempted contacts to potential study participants and we limited contacts accordingly to a single follow-up reminder at 8-10 weeks after first mailing. The subsequent response rates were approximately $25 \%$ with either 1 versus 3 mail out attempts. Potential participants' primary care physicians were notified of the study to ensure that there were no medical objections regarding participation; none objected. Due to resource constraints, questionnaires were available only in English. Individuals identified with Spanish surnames received an additional recruitment letter written in Spanish advising them to have an English-speaking family member to assist with translation of questionnaires.

\section{Study measures}

EOL care preferences were assessed using a previously published and validated instrument that generates seven dichotomous outcome variables related to EOL patient preferences. $[18,19]$ For this analysis, we focused on preferences regarding location of death and mechanical ventilation using a question stem that presented a hypothetical scenario of having an illness with certainty of $<1$ year to live. Assessment of preferred location of death was one item that asked if the illness got worse "where would you like to spend your last days-in a hospital, a nursing home, or at home?" Responses could be: "Hospital", "Nursing Home", "Home", "Don't know". "Home" and "Hospital" were examined for this study. Preferences regarding mechanical ventilation were assessed by combining two items which asked respondents whether they would use a mechanical ventilator for extending their life by 1 week or 1 month (yes, no, unknown). A 'Yes' response to either item was categorized as a preference for mechanical ventilation. Health literacy was assessed using the Newest Vital Sign (NVS), a 6-item health literacy assessment structured around reading and understanding information on a nutrition label. The NVS has been validated with high reliability and validity against the Rapid Assessment of health Literacy in Medicine (REALM) and the Short Test of Functional Literacy in Adults (STOFLHA) and has a high sensitivity for ascertainment of health literacy [20,21]. The scale classifies respondents' high likelihood of limited health literacy (score: 0-1), possibility of limited health literacy (score: $2-3$ ), and adequate health literacy (score: 4-6). For the purposes of this study, we condensed this to two categories: adequate health literacy (score $\geq 4$ ) or limited health literacy $($ score $<4)$ and those that did not fill out the scale 
were classified as "unknown”. Decisional self-efficacy was assessed using the Decisional Self-Efficacy Scale (DSES) an 11-item, 5-point rating scale with statements assessing a patient's feelings of adequacy and efficiency in dealing with life situations and perceived ability to engage in treatment decisions (score range: $0=$ "Not Confident", $2=$ "A Little Confident", to $4=$ "A Lot Confident"). The total score is summed, then divided by 11 and then multiplied by 25 , with scale score ranging from 0 (not confident) to 100 (extremely confident). The scale was developed by the Ottawa Hospital Research Institute Patient Decision Aids Research Group [22]. It provides a measure of a patient's ability to engage in procurement of information about treatment options, express concerns, and make treatment choices. The scale has a high reliability with a Cronbach's alpha coefficient of 0.84 .

Socio-demographic and cancer clinical characteristics were obtained from a combination of TCR registry data and self-reported information. Race/ethnicity was self-reported or, in cases of missing self-reported values, was obtained from TCR registry. Only 64 of 1460 respondents (4.4\%) did not self-report race/ethnicity and were thus assigned by the TCR data. Agreement between TCR race/ethnicity data for the remaining 1411 was high with $94 \%$ agreement on ethnicity and $98 \%$ agreement on race. Race and ethnicity groups are non-Hispanic white, non-Hispanic Black, and Hispanic. Additional items included age, gender, primary language spoken in the home, income, marital status, whether the individual lived alone or with others, and highest-attained education level. Self-reported health was collected using the Medicare Health Outcomes Survey [23], which asks respondents to rate their health on a 5-point scale from poor to excellent. Rurality and Texas Health Service Region were derived by zip code at the time of diagnosis of cancer, with rurality being defined per the US Department of Agriculture 2013 Rural/Urban Continuum Codes with scores of 1-3 yielding an urban designation and 4-9 being considered as rural [24]. Finally, cancer type and stage at diagnosis were obtained from the TCR database.

\section{Statistical analysis}

Descriptive statistics were generated for demographic and socio-economic characteristics. In order to address potential selection bias in this convenience sample, we performed an inverse probability weighting analysis [25]. For each observed case $(n=1460)$, we computed the probability of return of the survey form the pool of 5320 eligible non-missing cases. We determined the weights based upon five available factors: age, gender, race/ethnicity, cancer site, health services area in Texas. The inverse probability of response was derived to weight each observation attempting to balance the selection bias due to non-response. Finally, normalized inversed probability (inversed probability divided by the mean) was used in the final weighting analyses. Normalized inverse probability reduces the weight loading because weights can increase the standard errors of estimates and introduce instability in the data. Significance of differences between responses to items with regard to socio-demographic characteristics were assessed using a Rao-Scott Chisquare test for the weighted data [26]. Logistic regression analysis was conducted to examine the significance of demographic, socio-economic factors, health literacy and DSES score on respondents' preferences. All factors were included in the multivariable model, and odds ratios (OR), and 95\% confidence intervals (CI) were reported for each covariate, a $p$-value of $<0.05$ was deemed to be statistically significant. Finally, responses to the items were assessed using a one-way analysis of variance (ANOVA) test to examine the association of EOL preferences for location of death and use of mechanical ventilation with decisional self-efficacy using the DSES aggregate scores of each respondent. DSES score was included in the multivariable models as a continuous variable. Data analyses were performed using SAS (version 9.4 SAS Institute Inc., Cary, NC, USA).

\section{Results}

Of the 1566 respondents, 1460 (93.2\%) completed the EOL care, and decisional self-efficacy scale. Unweighted and weighted respondent characteristics are displayed in Table 1. In the unweighted sample, a majority of respondents were female (66.6\%), age 41-64 (51.1\%), married (65.8\%), college-educated $(73.9 \%)$, and reside within an urban location (86\%). A total of $72 \%$ of respondents were white compared to $10 \%$ black and $16 \%$ Hispanic. This is notably different from the racial make-up of Texas which is $41 \%$ non-Hispanic white, $40 \%$ Hispanic and $13 \%$ non-Hispanic black [27]. A majority of respondents reported an income level of $\$ 40,000-\$ 99,999$ (32.7\%) which is well above the reported median per capita income for Texans which is $\$ 30,143$ [27]. Most respondents (41.4\%) reported having "good" selfreported health, and had adequate health literacy (61.1\%). The most common primary disease sites were breast (39.5\%) and colorectal $(15.1 \%)$, with $57.8 \%$ of respondents having cancer localized to the primary site, $23.9 \%$ who had regional extension of their tumor and $7.2 \%$ with distant disease.

Among respondents, $7.6 \%$ expressed preference for dying at a hospital while $82.0 \%$ preferred to die at home. Distribution of respondent answers by socio-demographic factors by preference for death location is shown in Table 2. Univariate analyses revealed that $13.1 \%$ of Blacks and $13.6 \%$ of other (non-white, non-Hispanic) race respondents indicated a preference for spending their last day in the hospital. This finding remained significant on adjusted analyses (Table 3) which showed respondents who were Black $(\mathrm{OR}=1.80$; 
Table 1 Patient demographics

\begin{tabular}{|c|c|c|c|}
\hline & Number & Unweighted \% & Weighted \% \\
\hline Total cases & 1460 & 100.0 & 100.0 \\
\hline \multicolumn{4}{|l|}{ Age } \\
\hline $21-40$ & 102 & 7.0 & 7.5 \\
\hline $41-64$ & 746 & 51.1 & 50.6 \\
\hline $65-79$ & 547 & 37.5 & 36.9 \\
\hline $80+$ & 65 & 4.5 & 5.0 \\
\hline \multicolumn{4}{|l|}{ Gender } \\
\hline Female & 972 & 66.6 & 60.7 \\
\hline Male & 488 & 33.4 & 39.3 \\
\hline \multicolumn{4}{|l|}{ Race/ethnicity } \\
\hline White non-Hispanic & 1045 & 71.6 & 61.3 \\
\hline Black non-Hispanic & 144 & 9.9 & 13.2 \\
\hline Hispanic & 227 & 15.6 & 20.0 \\
\hline Others & 44 & 3.0 & 5.5 \\
\hline \multicolumn{4}{|l|}{ Language at home } \\
\hline English & 1311 & 89.8 & 86.1 \\
\hline Others & 100 & 6.9 & 9.7 \\
\hline UNK & 49 & 3.4 & 4.2 \\
\hline \multicolumn{4}{|l|}{ Education } \\
\hline$<$ Some HS & 104 & 7.1 & 9.3 \\
\hline HS & 251 & 17.2 & 19.2 \\
\hline Some college and above & 1079 & 73.9 & 69.6 \\
\hline UNK & 26 & 1.8 & 1.9 \\
\hline \multicolumn{4}{|l|}{ Marital status } \\
\hline Married & 961 & 65.8 & 65.1 \\
\hline Not married & 476 & 32.6 & 33.1 \\
\hline UNK & 23 & 1.6 & 1.9 \\
\hline \multicolumn{4}{|l|}{ Living arrangements } \\
\hline Alone & 253 & 17.3 & 16.6 \\
\hline With someone & 1190 & 81.5 & 82.0 \\
\hline UNK & 17 & 1.2 & 1.4 \\
\hline \multicolumn{4}{|l|}{ Income } \\
\hline Less than $\$ 19,999$ & 194 & 13.3 & 15.4 \\
\hline$\$ 20,000-\$ 39,999$ & 174 & 11.9 & 12.8 \\
\hline$\$ 40,000-\$ 99,999$ & 478 & 32.7 & 32.0 \\
\hline$\$ 100,000$ or more & 393 & 26.9 & 24.4 \\
\hline UNK & 221 & 15.1 & 15.3 \\
\hline \multicolumn{4}{|l|}{ Rural/urban } \\
\hline Urban & 1256 & 86.0 & 85.8 \\
\hline Rural & 204 & 14.0 & 14.2 \\
\hline \multicolumn{4}{|l|}{ Self-reported health } \\
\hline Excellent & 479 & 32.8 & 31.2 \\
\hline Good & 604 & 41.4 & 40.5 \\
\hline Fair & 289 & 19.8 & 21.6 \\
\hline Poor & 81 & 5.6 & 6.3 \\
\hline \multicolumn{4}{|l|}{ Health literacy score } \\
\hline Limited literacy $(<=4)$ & 439 & 30.1 & 32.9 \\
\hline Adequate literacy $(>4)$ & 892 & 61.1 & 57.3 \\
\hline UNK & 129 & 8.8 & 9.8 \\
\hline
\end{tabular}

$H S$, high school; $U N K$, unknown
95\% CI: 1.19-2.73, $p=0.005)$ or of other non-white race/ ethnicity (OR $=3.53 ; 95 \% \mathrm{CI}: 1.99-6.27, p<0.001)$ were significantly and independently more likely to indicate preference for spending their last days in a hospital compared to white respondents. Preference for dying at home was observed among male respondents, those with college education or greater, and those with poor self-reported health. On multivariable analysis (Table 3 ), those with college education or greater $(\mathrm{OR}=0.40 ; 95 \% \mathrm{CI}: 0.24-0.67, p<0.001)$, non-English language spoken at home $(\mathrm{OR}=0.54 ; 95 \% \mathrm{CI}$ : $0.30-0.98, p=0.04$ ), and having poor self-reported health $(\mathrm{OR}=0.41 ; 95 \%$ CI: $0.18-0.97, p=0.04)$ were independently associated with less likelihood for indicating preference for dying in a hospital.

Univariate analyses (Table 2) further revealed that gender $(p=0.024)$, education level $(p=0.033)$, and self-reported health $(p=0.021)$ were significantly associated with preference to die at home. On adjusted analyses male gender ( $\mathrm{OR}=1.48$; 95\% CI: 1.19-1.85, $p=0.0 .001)$, college education or greater $(\mathrm{OR}=2.25 ; 95 \% \mathrm{CI}: 1.51-3.36, p<0.001)$, and poor (vs excellent) self-reported health $(\mathrm{OR}=1.92$; 95\%CI: $1.10-3.34, p=0.021)$ were independently associated with increased likelihood of preference for dying at home. Factors that were independently associated with a decreased likelihood of preferring death at home were all age categories greater than 40 years (compared to ages $21-40$ years $)$, Other non-white race/ethnicity $(\mathrm{OR}=0.47$; 95\%CI: $0.30-0.73, p=0.001)$; unmarried status $(\mathrm{OR}=0.66$; 95\% CI: $0.50-0.87, p=0.003$ ), and good (vs excellent) selfreported health (OR $=0.77$; 95\% CI: $0.60-0.99, p=0.040)$. Notably, DSES were not associated with EOL preferences. Mean DSES scores were 84.8 [standard deviation (SD) 18.5), 85.1 (SD 18.4), and 85.4 (SD 19.1) for those exhibiting preferences to die at the hospital, die at home and for mechanical ventilation, respectively.

Investigation of preferences regarding use of mechanical ventilation at the EOL showed $25.3 \%$ of respondents reported a preference for mechanical ventilation while $74.7 \%$ preferred no mechanical ventilation. Table 4 shows univariate analysis revealing that age, race/ethnicity, educational attainment, language spoken at home, and health literacy score were significantly associated with preferences regarding mechanical ventilation. In adjusted analyses, two factors were independently associated with higher likelihood of preference for undergoing mechanical ventilation at the EOL: race/ethnicity and living with someone vs. alone. Specifically, Black OR $=2.25(95 \% \mathrm{CI} ; 1.71-2.95, p<0.001)$, Hispanic OR $=1.61(95 \% \mathrm{CI} ; 1.23-2.11, p=0.001)$, and other non-white race/ethnicity (vs white race/ethnicity) $\mathrm{OR}=1.79(95 \% \mathrm{CI} ; 1.19-2.71, p=0.005)$ all were more likely to prefer mechanical ventilation compared to white respondents. Those living with someone else (vs. living alone) $(\mathrm{OR}=1.60 ; 95 \% \mathrm{CI}$ : $1.15-2.21, p=0.005)$ were also 
Table 2 Respondent answers (weighted) to patients preference for location of death by socio-economic factors

\begin{tabular}{|c|c|c|c|c|}
\hline & $\begin{array}{l}\text { Preference dying at } \\
\text { hospital (\%) }\end{array}$ & $\begin{array}{l}\text { Rao-Scott } \\
p \text {-value }\end{array}$ & $\begin{array}{l}\text { Preference for dying at } \\
\text { home }(\%)\end{array}$ & $\begin{array}{l}\text { Rao-Scott } \\
p \text {-value }\end{array}$ \\
\hline Total cases & 107 (7.6) & & $1198(82.0)$ & \\
\hline Gender & & 0.652 & & 0.024 \\
\hline Female & $75(7.9)$ & & 783 (79.9) & \\
\hline Male & $32(7.1)$ & & $415(85.3)$ & \\
\hline Age & & 0.816 & & 0.111 \\
\hline $21-40$ & $*(6.0)$ & & $91(90.1)$ & \\
\hline $41-64$ & $60(8.2)$ & & $620(82.7)$ & \\
\hline $65-79$ & $33(7.0)$ & & 437 (79.7) & \\
\hline $80+$ & $*(7.9)$ & & $50(80.5)$ & \\
\hline Race/ethnicity & & 0.012 & & 0.071 \\
\hline White & $65(5.8)$ & & $861(83.2)$ & \\
\hline Black & $18(13.1)$ & & $111(75.8)$ & \\
\hline Hispanic & $18(7.7)$ & & $193(85.0)$ & \\
\hline Other & $*(13.6)$ & & 33 (73.6) & \\
\hline Education & & 0.096 & & 0.033 \\
\hline$\leq$ Some HS & $14(13.4)$ & & $79(76.8)$ & \\
\hline HS & $22(9.7)$ & & $194(76.1)$ & \\
\hline College or $>$ & $70(6.2)$ & & 903 (84.4) & \\
\hline Income & & 0.221 & & 0.100 \\
\hline$\leq \$ 19.9 \mathrm{~K}$ & $13(8.7)$ & & $163(83.2)$ & \\
\hline$\$ 20 \mathrm{~K}-\$ 39.9 \mathrm{~K}$ & $*(7.5)$ & & 145 (81.7) & \\
\hline$\$ 40 \mathrm{~K}-\$ 99.9 \mathrm{~K}$ & $40(7.4)$ & & $389(83.2)$ & \\
\hline$\geq \$ 100,000$ & $23(5.0)$ & & $333(84.5)$ & \\
\hline UNK & $21(11.1)$ & & $168(74.7)$ & \\
\hline Marital status & & 0.470 & & 0.192 \\
\hline Married & $66(6.8)$ & & $803(83.5)$ & \\
\hline Not married & $40(9.1)$ & & 375 (78.9) & \\
\hline Living situation & & 0.750 & & 0.972 \\
\hline Alone & $21(7.9)$ & & $204(81.4)$ & \\
\hline With someone & $85(7.4)$ & & $979(82.1)$ & \\
\hline Home language & & 0.937 & & 0.862 \\
\hline English & 98 (7.6) & & $1072(81.9)$ & \\
\hline Others & $*(8.1)$ & & $83(81.5)$ & \\
\hline UNK & $*(6.1)$ & & $43(85.4)$ & \\
\hline Rural/urban & & 0.641 & & 0.422 \\
\hline Urban & $93(7.7)$ & & $1031(81.7)$ & \\
\hline Rural & $14(6.7)$ & & $167(84.1)$ & \\
\hline Self-reported health & & 0.637 & & 0.021 \\
\hline Excellent & $34(6.8)$ & & $404(84.8)$ & \\
\hline Good & $45(8.3)$ & & $485(79.4)$ & \\
\hline Fair & $22(8.2)$ & & $238(81.5)$ & \\
\hline Poor & $*(4.2)$ & & $68(89.0)$ & \\
\hline Health literacy & & 0.050 & & 0.109 \\
\hline Limited $(\leq 4)$ & $39(9.2)$ & & $352(79.8)$ & \\
\hline Adequate $(>4)$ & $57(5.9)$ & & $742(84.1)$ & \\
\hline UNK & $11(11.9)$ & & $104(77.4)$ & \\
\hline
\end{tabular}

$H S$, high school; $K$, thousand US dollars; $U N K$, unknown; * Raw cell number counts surpressed to preserve anonymity and comply with data user agreement with TCR 
Table 3 Weighted multivariate analyses of patients preferences for place of death and mechanical ventilation

\begin{tabular}{|c|c|c|c|c|}
\hline \multicolumn{5}{|l|}{ Multivariate analysis } \\
\hline \multirow[t]{2}{*}{ Factor } & \multirow[t]{2}{*}{ Reference group } & Preference to die at hospital & Preference to die at home & $\begin{array}{l}\text { Preference for mechani- } \\
\text { cal ventilation at EOL }\end{array}$ \\
\hline & & OR $(95 \% \mathrm{CI}) p$-value & OR $(95 \% \mathrm{CI}) p$-value & OR $(95 \% \mathrm{CI}) p$-value \\
\hline \multicolumn{5}{|l|}{ Gender } \\
\hline Male & Female & $0.90(0.66-1.24) 0.524$ & $1.48(1.19-1.85) 0.001$ & 1.09 (0.90-1.33) 0.374 \\
\hline \multicolumn{5}{|l|}{ Age } \\
\hline $41-64$ & $21-40$ & $1.43(0.75-2.73) 0.284$ & $0.49(0.29-0.81) 0.005$ & $0.54(0.39-0.75)<0.001$ \\
\hline $65-79$ & & $1.16(0.59-2.27) 0.668$ & $0.38(0.23-0.64)<.001$ & $0.36(0.25-0.51)<0.001$ \\
\hline $80+$ & & $1.25(0.50-3.13) 0.642$ & $0.40(0.20-0.78) 0.008$ & $0.30(0.17-0.53)<0.001$ \\
\hline \multicolumn{5}{|l|}{ Race/ethnicity } \\
\hline Black & White & $1.80(1.19-2.73) 0.005$ & $0.74(0.55-1.01) 0.061$ & $2.25(1.71-2.95)<0.001$ \\
\hline Hispanic & & $1.27(0.81-1.98) 0.296$ & $1.27(0.91-1.78) 0.156$ & $1.61(1.23-2.11) 0.001$ \\
\hline Other & & $3.53(1.99-6.27)<.001$ & $0.47(0.30-0.73) 0.001$ & 1.79 (1.19-2.71) 0.005 \\
\hline \multicolumn{5}{|l|}{ Education } \\
\hline HS & $<$ Some HS & $0.60(0.35-1.01) 0.052$ & $1.22(0.81-1.83) 0.340$ & $0.48(0.34-0.70)<0.001$ \\
\hline College or $>$ & & $0.40(0.24-0.67)<.001$ & $2.25(1.51-3.36)<.001$ & $0.50(0.35-0.70) 0.001$ \\
\hline UNK & & $0.44(0.08-2.46) 0.352$ & $1.19(0.41-3.45) 0.756$ & 1.59 (0.62-4.08) 0.332 \\
\hline \multicolumn{5}{|l|}{ Income } \\
\hline$\$ 20 \mathrm{~K}-\$ 39.9 \mathrm{~K}$ & $<\$ 19.9 \mathrm{~K}$ & $1.06(0.60-1.86) 0.844$ & $0.75(0.50-1.12) 0.157$ & $1.43(1.01-2.02) 0.043$ \\
\hline$\$ 40 \mathrm{~K}-\$ 99.9 \mathrm{~K}$ & & $1.26(0.76-2.08) 0.375$ & $0.73(0.51-1.06) 0.098$ & $0.96(0.69-1.32) 0.794$ \\
\hline$\geq \$ 100,000$ & & $0.85(0.47-1.55) 0.591$ & $0.68(0.45-1.03) 0.067$ & $0.91(0.63-1.31) 0.607$ \\
\hline UNK & & $1.44(0.86-2.42) 0.168$ & $0.55(0.38-0.81) 0.002$ & $1.14(0.80-1.62) 0.457$ \\
\hline \multicolumn{5}{|l|}{ Marital status } \\
\hline Not married & Married & $1.26(0.86-1.86) 0.242$ & $0.66(0.50-0.87) 0.003$ & $1.18(0.92-1.51) 0.196$ \\
\hline UNK & & $1.16(0.28-4.73) 0.837$ & $1.14(0.39-3.35) 0.819$ & $0.68(0.28-1.67) 0.399$ \\
\hline \multicolumn{5}{|l|}{ Living situation } \\
\hline With someone & Alone & $1.18(0.72-1.93) 0.505$ & $0.73(0.52-1.03) 0.070$ & $1.60(1.15-2.21) 0.005$ \\
\hline UNK & & $2.63(0.50-14.0) 0.256$ & $1.27(0.35-4.61) 0.720$ & $1.36(0.44-4.20) 0.592$ \\
\hline \multicolumn{5}{|l|}{ Home language } \\
\hline Others & English & $0.54(0.30-0.98) 0.041$ & $1.25(0.81-1.92) 0.316$ & $1.00(0.70-1.43) 0.985$ \\
\hline UNK & & $0.42(0.15-1.14) 0.088$ & $1.33(0.70-2.55) 0.385$ & $0.97(0.59-1.61) 0.909$ \\
\hline \multicolumn{5}{|l|}{ Rural/urban } \\
\hline Rural & Urban & $0.85(0.54-1.35) 0.491$ & $1.20(0.88-1.64) 0.256$ & $0.83(0.63-1.11) 0.215$ \\
\hline \multicolumn{5}{|l|}{ Self-reported health } \\
\hline Good & Excellent & $1.09(0.76-1.57) 0.641$ & $0.77(0.60-0.99) 0.040$ & $0.76(0.61-0.94) 0.013$ \\
\hline Fair & & $1.03(0.67-1.59) 0.887$ & $0.94(0.69-1.27) 0.681$ & $0.77(0.59-1.00) 0.054$ \\
\hline Poor & & $0.41(0.18-0.97) 0.042$ & $1.92(1.10-3.34) 0.021$ & $0.73(0.48-1.12) 0.150$ \\
\hline Unknown & & $2.60(0.38-17.6) 0.328$ & $0.18(0.05-0.60) 0.005$ & \\
\hline Health literacy & Limited $(\leq 4)$ & & & \\
\hline Adequate $(>4)$ & & $0.75(0.53-1.06) 0.103$ & $1.18(0.93-1.49) 0.186$ & $0.80(0.65-0.99) 0.040$ \\
\hline UNK & & $1.05(0.65-1.70) 0.836$ & $1.06(0.74-1.52) 0.739$ & $0.80(0.57-1.13) 0.207$ \\
\hline DSE score & Continuous & $1.00(0.99-1.01) 0.790$ & 1.00 (1.00-1.01) 0.436 & $1.00(1.00-1.01) 0.885$ \\
\hline
\end{tabular}

$H S$, high school; $K$, thousand US Dollars; UNK, unknown; DSE, decisional self-efficacy

more likely to prefer mechanical ventilation at the EOL. The following were independently associated with lower likelihood of preference for mechanical ventilation: older age versus age $21-40$ years $(p<0.001)$, higher educational levels such as completion of high school $(\mathrm{OR}=0.48 ; 95 \% \mathrm{CI}$ :
0.34-0.70, $p<0.001$ ) and $\geq$ college education (vs $\leq$ some high school) $(\mathrm{OR}=0.50(95 \% \mathrm{CI} ; 0.35-0.70, p=0.001)$, good (vs excellent) self-reported health $(\mathrm{OR}=0.76$; $95 \% \mathrm{CI}$ : $0.61-0.94, p=0.013)$ and adequate (vs limited) health literacy $(\mathrm{OR}=0.80$; 95\% CI: $0.65-0.99, p=0.040)$. 
Table 4 Respondent answers (weighted) to patients preference for mechanical ventilation at EOL by socio-economic factors

\begin{tabular}{|c|c|c|}
\hline & $\begin{array}{l}\text { Yes to Ventilator at EOL } \\
(\%)\end{array}$ & $\begin{array}{l}\text { Rao-Scott } \\
p \text {-value }\end{array}$ \\
\hline Total cases & $340(25.3)$ & \\
\hline Gender & & 0.992 \\
\hline Female & $218(25.3)$ & \\
\hline Male & $122(25.3)$ & \\
\hline Age & & $<.001$ \\
\hline $21-40$ & $42(42.5)$ & \\
\hline $41-64$ & $180(27.8)$ & \\
\hline $65-79$ & $107(19.6)$ & \\
\hline $80+$ & $11(16.0)$ & \\
\hline Race/ethnicity & & $<.001$ \\
\hline White & $191(18.7)$ & \\
\hline Black & $58(38.9)$ & \\
\hline Hispanic & $77(34.7)$ & \\
\hline Other & $14(31.3)$ & \\
\hline Education & & $<.001$ \\
\hline$<$ Some HS & $42(42.8)$ & \\
\hline HS & $56(24.6)$ & \\
\hline College or $>$ & $233(22.7)$ & \\
\hline Income & & 0.062 \\
\hline$\leq \$ 19.9 \mathrm{~K}$ & $52(27.9)$ & \\
\hline$\$ 20 \mathrm{~K}-\$ 39.9 \mathrm{~K}$ & $50(32.2)$ & \\
\hline$\$ 40 \mathrm{~K}-\$ 99.9 \mathrm{~K}$ & $101(21.7)$ & \\
\hline$\geq \$ 100,000$ & $84(22.5)$ & \\
\hline UNK & $53(28.8)$ & \\
\hline Marital status & & 0.469 \\
\hline Married & $216(24.2)$ & \\
\hline Not married & $117(27.1)$ & \\
\hline Living situation & & 0.082 \\
\hline Alone & 48 (19.4) & \\
\hline With someone & $286(26.3)$ & \\
\hline Home language & & 0.016 \\
\hline English & $286(23.6)$ & \\
\hline Others & $38(35.9)$ & \\
\hline UNK & $16(34.6)$ & \\
\hline Rural/urban & & 0.204 \\
\hline Urban & $300(26.0)$ & \\
\hline Rural & $40(21.2)$ & \\
\hline Self-reported health & & 0.667 \\
\hline Excellent & $120(27.6)$ & \\
\hline Good & $131(23.7)$ & \\
\hline Fair & $70(25.5)$ & \\
\hline Poor & $19(25.1)$ & \\
\hline Health literacy & & 0.032 \\
\hline Limited $(\leq 4)$ & $121(30.0)$ & \\
\hline Adequate $(>4)$ & $191(22.4)$ & \\
\hline UNK & $28(26.5)$ & \\
\hline
\end{tabular}

$E O L$, end of life; $H S$, high school; $K$, thousand US dollars; $U N K$, unknown

\section{Discussion}

In this population-based survey study of Texas cancer patients, we observed that non-white individuals recently diagnosed with cancer were more likely to express preferences for higher acuity EOL care, such as spending their last days in the hospital or desiring mechanical ventilation, compared to their white counterparts. These findings persisted even when adjusting for socio-demographic variables, health literacy, and decisional self-efficacy. These observations are of particular relevance given a prior study showing that Black and Hispanic Texas cancer patients were more likely to receive more aggressive care at the EOL[4] and a subsequent study showing that hospice use was significantly lower among non-white cancer patients in Texas [5]. The latter study attempted to ascertain whether payer benefit characteristics influenced hospice utilization and found that even though differences in hospice utilization previously observed between fee-for-service Medicare and Medicare managed care were eliminated over time, racial/ethnic disparities in hospice use persisted. Our study suggests that racial/ethnic variation in EOL care may be a result of preferences among non-white patients for more aggressive care.

Other studies have reported that non-white patients express preferences towards aggressive EOL care measures [18, 28, 29]. A survey on over 2500 Medicare patients evaluating patients willingness to engage in various EOL treatment measures noted that $35 \%$ of Black vs $15 \%$ of white patients expressed a desire for more treatment [29]. A systematic review examined the impact of ethnic disparities on EOL care preferences and reported that Black patients preferred more aggressive treatment and that both Black and Hispanic patients were less likely to have documented advanced care plans [30]. The latter observation was at least partially attributable to religious coping and spirituality concerns which were not measured in our study [30]. Another systematic review similarly showed that Blacks preferred aggressive EOL care citing knowledge of and access to hospice services as one of the potential explanations [15]. Others have reported Black and Hispanic patients' lack of awareness or information about hospice services [31, 32]. Inadequate access to hospice potentially hampering high-quality EOL care and "good death"[7] is a concern of national importance. A recently published analysis of over 125,000 Medicare beneficiaries treated at NCI/NCCN cancer centers showing that cancer centers serving primarily non-white patients independently had inferior performance on EOL quality metrics translating to more aggressive EOL measures including ICU admissions and lack of hospice referral [3].

Place of death may serve as a surrogate for aggressiveness of care as death within a hospital can correspond with 
decreased participation in hospice services. Data reveal that a majority (50-90\%) of cancer patients express a preference for dying at home [33]. Despite this, there has been a growing trend toward cancer deaths occurring away from home [34]. If individuals prefer death out of a hospital, this trend suggests EOL care that may be discordant with preferences. However, even among patients who may prefer death in the hospital, this setting for death is associated with elevated health care costs and, more importantly, poor quality of life for patients and families [4, 8-10]. Investigators have shown that cancer patients who die in an ICU or hospital experience more physical and emotional distress and worse quality of life near death $[35,36]$. Furthermore, caregivers of patients who die in the hospital or ICU have heightened risk of post-traumatic stress disorder from the EOL experience of their family member [35]. Thus, cancer-related death that includes high-acuity care have negative impacts for both patients and subsequently bereaved caregivers.

Our study offers data to aid in identifying specific patient groups that may gravitate towards aggressive EOL care measures. Such information could help clinicians and policy makers craft programs and policies tailored to groups at risk of poor quality EOL care. Possible rationale for such preferences among non-white patients may involve strong religious beliefs, family/social dynamics, or significant barriers to access of palliative care services for such patients in general [37-42]. It would likely serve to improve palliative care provision if providers were attuned to which patients may elect more aggressive EOL care and engage in early discussions regarding potential benefits or drawbacks to aggressive care and thus facilitate advanced care planning. Furthermore, evidence suggests that performing advanced care planning and discussing preferences regarding location of death will increase the probability of patients dying at their preferred location [43].

Culturally competent and patient-centered palliative care programs improve EOL care quality among racial/ ethnic minority populations $[44,45]$. The observation in our study that adequate health literacy was independently associated with a decreased likelihood of preference for mechanical ventilation at the EOL suggests that there is some quality of care improvement to be affected by welldesigned, culturally responsive efforts to educate diverse cancer patients about the expected benefits of aggressive EOL care measures. Additional factors beyond race/ ethnicity contribute to EOL care preferences. We also observed that an individual's home social situation influenced preferences regarding aggressiveness of EOL care. Unmarried individuals were less likely to want to die at home, possibly owing to not wanting to be alone at the EOL. This raises the importance of assessment of social supports for individuals with terminal cancer. Respondents who lived with others versus alone were more likely to express favorable preferences for mechanical ventilation use, perhaps reflecting that the aggressive measures may stave off abandonment of family members should they become terminally ill. Awareness of this fact can help individuals cope with EOL decision-making relative to a terminally ill patient's relationships and feelings of responsibility. This highlights the fact that while valuable, race/ ethnicity remains a complex concept influenced by several additional factors including culture, socioeconomic status, and personal values thus conditioning the conclusions that can be drawn and generalizability of race/ethnicity-specific data [18].

It is important to acknowledge several limitations inherent to this mail-in survey study. These include non-response bias, low response rate, questionable comprehension capabilities for individuals with low health literacy or those who are non-English speakers, and the inability to question respondents for further information/rationale for their responses. Unfortunately due to resource limitations, our study was unable to provide a Spanish version of this survey, thus explaining the under-representative of non-English speaking Hispanic patients in Texas [27]. The use of a relative to assist with translation of the survey for Spanish speakers may have also resulted in inaccurate translations and corresponding responses. Additionally, there was significant overrepresentation of non-Hispanic respondents relative to the state population which impacts the generalizability of this data. Additionally, some investigators have shown that preferences regarding EOL care interventions may vary over the time course of a life-threatening disease such that, as a patient experiences more disability from disease or expected disability from more treatment, they may correspondingly desire less aggressive care [46]. We only surveyed patients at one time point in their cancer disease course and thus did not capture this potential phenomenon. Despite these limitations, considering the large size of this study and important findings, we feel that it contributes to our understanding of EOL care preferences in cancer patients among a large, populous state whose demographic make-up mirrors the expected future diversity of the US national population.

In conclusion, non-white racial/ethnic minorities recently diagnosed with cancer expressed generally more favorable preferences towards pursuit of aggressive EOL measures including dying in a hospital and pursuing mechanical ventilation. These findings suggest that observed disparities reported regarding potential low quality EOL care among non-white patients with cancer is, at least in part, a reflection of patient preferences. These data may help inform programmatic or policy initiatives to address observed disparities and ensure that members of all racial/ethnic groups have access to their preferred "good death". 
Author contribution Conceptualization: B. Ashleigh Guadagnolo. Statistical analysis: Kaiping Liao. Composition/writing of initial draftDavid Boyce-Fappiano, B. Ashleigh Guadagnolo. Reviewing \& Editing: David Boyce-Fappiano, Kaiping Liao, Christopher Miller, Susan K. Peterson, Linda Elting, B. Ashleigh Guadagnolo.

Funding This research was supported by a grant from the Cancer Prevention Research Institute of Texas (RP160674, Guadagnolo co-PI). The funding source was uninvolved in the conduct of the research and the interpretation of results.

Data availability The authors have full control of the data which were obtained under a Data Use Agreement from the Texas Cancer Registry and the Centers for Medicare and Medicaid Services. These data are confidential and cannot be shared. The TCR is the 4th largest cancer registry in the US that meets the rigorous data standards of the National Program of the Central Cancer Registries and the Centers for Disease Control, and is Gold Certified by the North American Association of Central Cancer Registries.

\section{Declarations}

Ethics approval and consent to participate All study procedures were approved by both the Texas Department of State Health Services and MD Anderson institutional review boards. All subjects voluntarily participated in this study.

Consent for publication All subjects voluntarily participated in this study and authorized MD Anderson Cancer Center to utilize and share the obtained data for research and publication purposes.

Conflict of interest The authors declare no conflicts of interest with the funder and none with other entities related to the research.

\section{References}

1. Earle CC, Neville BA, Landrum MB, Ayanian JZ, Block SD, Weeks JC (2004) Trends in the aggressiveness of cancer care near the end of life. J Clin Oncol Off J Am Soc Clin Oncol 22(2):315321. https://doi.org/10.1200/JCO.2004.08.136

2. Wright AA, Hatfield LA, Earle CC, Keating NL (2014) End-oflife care for older patients with ovarian cancer is intensive despite high rates of hospice use. J Clin Oncol Off J Am Soc Clin Oncol 32(31):3534-3539. https://doi.org/10.1200/JCO.2014.55.5383

3. Wasp GT, Alam SS, Brooks GA et al (2020) End-of-life quality metrics among medicare decedents at minority-serving cancer centers: a retrospective study. Cancer Med 9(5):1911-1921. https://doi.org/10.1002/cam4.2752

4. Guadagnolo BA, Liao K-P, Giordano SH, Elting LS, Shih Y-CT (2015) Variation in intensity and costs of care by payer and race for patients dying of cancer in Texas: an analysis of Registrylinked Medicaid, Medicare, and Dually Eligible Claims Data. Med Care. 53(7):591-598. https://doi.org/10.1097/MLR.0000000000 000369

5. Elting LS, Liao K-P, Giordano SH, Guadagnolo BA (2020) Hospice enrollment among cancer patients in Texas covered by Medicare managed care and traditional fee-for-service plans: a statewide population-based study. Support Care Cancer Off J Multinatl Assoc Support Care Cancer 28(7):3351-3359. https://doi.org/10. 1007/s00520-019-05142-z
6. Earle CC, Landrum MB, Souza JM, Neville BA, Weeks JC, Ayanian JZ (2008) Aggressiveness of cancer care near the end of life: is it a quality-of-care issue? J Clin Oncol 26(23):3860 3866. https://doi.org/10.1200/JCO.2007.15.8253

7. Institute of Medicine (IOM) (1997) Committee on care at the end of life, division of health, care services. Approaching death, improving care at the end of life. In: Field M, Cassel C (eds). National Academy Press, pp 23-25

8. Wright AA, Zhang B, Ray A et al (2008) Associations between end-of-life discussions, patient mental health, medical care near death, and caregiver bereavement adjustment. JAMA J Am Med Assoc 300(14):1665-1673. https://doi.org/10.1001/jama.300. 14.1665

9. Chastek B, Harley C, Kallich J, Newcomer L, Paoli CJ, Teitelbaum AH (2012) Health care costs for patients with cancer at the end of life. J Oncol Pract 8(6S):75s-80s. https://doi.org/10. 1200/JOP.2011.000469

10. Round J, Jones L, Morris S (2015) Estimating the cost of caring for people with cancer at the end of life: A modelling study. Palliat Med 29(10):899-907. https://doi.org/10.1177/0269216315 595203

11. Lunney JR, Lynn J, Foley DJ, Lipson S, Guralnik JM (2003) Patterns of functional decline at the end of life. JAMA 289(18):23872392. https://doi.org/10.1001/jama.289.18.2387

12. Teno JM, Weitzen S, Fennell ML, Mor V (2001) Dying trajectory in the last year of life: does cancer trajectory fit other diseases? J Palliat Med 4(4):457-464. https://doi.org/10.1089/1096621017 53381593

13. Mack JW, Weeks JC, Wright AA, Block SD, Prigerson HG (2010) End-of-life discussions, goal attainment, and distress at the end of life: predictors and outcomes of receipt of care consistent with preferences. J Clin Oncol 28(7):1203-1208. https://doi.org/10. 1200/JCO.2009.25.4672

14. Ahluwalia SC, Tisnado DM, Walling AM et al (2015) Association of early patient-physician care planning discussions and end-oflife care intensity in advanced cancer. J Palliat Med 18(10):834841. https://doi.org/10.1089/jpm.2014.0431

15. Wicher CP, Meeker MA (2012) What influences African American end-of-life preferences? J Health Care Poor Underserved 23(1):28-58. https://doi.org/10.1353/hpu.2012.0027

16. Current Projects I Sealy Center on Aging I UTMB Home. https:// www.utmb.edu/scoa/research/supported-research-programs/ comparative-effectiveness-research-on-cancer-in-texas/currentprojects. Accessed 14 Jan 2021

17. TCR I Home. Accessed September 3, 2020. https://www.dshs. state.tx.us/tcr/. Accessed 14 Jan 2021

18. Barnato AE, Anthony DL, Skinner J, Gallagher PM, Fisher ES (2009) Racial and ethnic differences in preferences for end-of-life treatment. J Gen Intern Med 24(6):695-701. https://doi.org/10. 1007/s11606-009-0952-6

19. Barnato AE, Herndon MB, Anthony DL et al (2007) Are regional variations in end-of-life care intensity explained by patient preferences? Med Care 45(5):386-393. https://doi.org/10.1097/01.mlr. 0000255248.79308 .41

20. Weiss BD, Mays MZ, Martz W et al (2005) Quick assessment of literacy in primary care: the newest vital sign. Ann Fam Med 3(6):514-522. https://doi.org/10.1370/afm.405

21. Osborn CY, Weiss BD, Davis TC et al (2007) Measuring adult literacy in health care: performance of the newest vital sign. Am J Health Behav 31(Suppl 1):S36-46. https://doi.org/10.5555/ajhb. 2007.31.supp.S36

22. O'Connor A. User Manual-Decision Self-Efficacy Scale [Internet]. Published modified 2002 1995. Accessed November 16, 2020. https://decisionaid.ohri.ca/docs/develop/User_Manuals/ UM_Decision_SelfEfficacy.pdf. Accessed 15 Aug 2020 
23. Medicare Health Outcomes Survey. https://www.hosonline.org/. Accessed 15 Aug 2020

24. USDA ERS - Rural-Urban Continuum Codes. Accessed September 3, 2020. https://www.ers.usda.gov/data-products/rural-urbancontinuum-codes.aspx. Accessed 5 Dec 2020

25. Seaman SR, White IR (2013) Review of inverse probability weighting for dealing with missing data. Stat Methods Med Res 22(3):278-295. https://doi.org/10.1177/0962280210395740

26. Rao JNK, Scott AJ (1987) On simple adjustments to chi-square tests with sample survey data. Ann Stat 15(1):385-397. https:// doi.org/10.1214/aos/1176350273

27. U.S. Census Bureau QuickFacts: Texas. Accessed August 31, 2020. https://www.census.gov/quickfacts/TX

28. Caralis PV, Davis B, Wright K, Marcial E (1993) The influence of ethnicity and race on attitudes toward advance directives, lifeprolonging treatments, and euthanasia. J Clin Ethics 4(2):155-165

29. Garrett JM, Harris RP, Norburn JK, Patrick DL, Danis M (1993) Life-sustaining treatments during terminal illness: who wants what? J Gen Intern Med 8(7):361-368

30. LoPresti MA, Dement F, Gold HT (2016) End-of-life care for people with cancer from ethnic minority groups: a systematic review. Am J Hosp Palliat Care 33(3):291-305. https://doi.org/10.1177/ 1049909114565658

31. Rhodes RL, Teno JM, Welch LC (2006) Access to hospice for African Americans: are they informed about the option of hospice? J Palliat Med 9(2):268-272. https://doi.org/10.1089/jpm. 2006.9.268

32. Born W, Greiner KA, Sylvia E, Butler J, Ahluwalia JS (2004) Knowledge, attitudes, and beliefs about end-of-life care among inner-city African Americans and Latinos. J Palliat Med 7(2):247256. https://doi.org/10.1089/109662104773709369

33. Higginson IJ, Sen-Gupta GJ (2000) Place of care in advanced cancer: a qualitative systematic literature review of patient preferences. J Palliat Med 3(3):287-300. https://doi.org/10.1089/jpm. 2000.3.287

34. Munday D, Dale J, Murray S (2007) Choice and place of death: individual preferences, uncertainty, and the availability of care. $\mathrm{J}$ R Soc Med 100(5):211-215

35. Wright AA, Keating NL, Balboni TA, Matulonis UA, Block SD, Prigerson HG (2010) Place of death: correlations with quality of life of patients with cancer and predictors of bereaved caregivers' mental health. J Clin Oncol Off J Am Soc Clin Oncol 28(29):4457-4464. https://doi.org/10.1200/JCO.2009.26.3863

36. Roulston A, Campbell A, Cairnduff V, Fitzpatrick D, Donnelly C, Gavin A (2017) Bereavement outcomes: a quantitative survey identifying risk factors in informal carers bereaved through cancer. Palliat Med 31(2):162-170. https://doi.org/10.1177/02692 16316649127

37. Phelps AC, Maciejewski PK, Nilsson M et al (2009) Religious coping and use of intensive life-prolonging care near death in patients with advanced cancer. JAMA 301(11):1140-1147. https:// doi.org/10.1001/jama.2009.341

38. Crawley L (2000) Palliative and end-of-life care in the African American Community. JAMA 284(19):2518. https://doi.org/10. 1001/jama.284.19.2518

39. Gordon AK (1996) Hospice and minorities: a national study of organizational access and practice. Hosp J 11(1):49-70. https:// doi.org/10.1080/0742-969X.1996.11882815

40. Morrison RS, Zayas LH, Mulvihill M, Baskin SA, Meier DE (1998) Barriers to completion of health care proxies: an examination of ethnic differences. Arch Intern Med 158(22):2493. https:// doi.org/10.1001/archinte.158.22.2493

41. Johnson KS (2013) Racial and ethnic disparities in palliative care. J Palliat Med 16(11):1329-1334. https://doi.org/10.1089/ jpm.2013.9468

42. Yarnell CJ, Fu L, Bonares MJ, Nayfeh A, Fowler RA (2020) Association between Chinese or South Asian ethnicity and end-of-life care in Ontario, Canada. CMAJ Can Med Assoc J 192(11):E266E274. https://doi.org/10.1503/cmaj.190655

43. Collis E, Al-Qurainy R (2013) Care of the dying patient in the community. BMJ 347:f4085. https://doi.org/10.1136/bmj.f4085

44. Fischer SM, Sauaia A, Kutner JS (2007) Patient navigation: a culturally competent strategy to address disparities in palliative care. J Palliat Med 10(5):1023-1028. https://doi.org/10.1089/jpm. 2007.0070

45. Marr L, Neale D, Wolfe V, Kitzes J (2012) Confronting myths: the Native American experience in an academic inpatient palliative care consultation program. J Palliat Med 15(1):71-76. https://doi. org/10.1089/jpm.2011.0197

46. Fried TR, Bradley EH, Towle VR, Allore H (2002) Understanding the treatment preferences of seriously ill patients. N Engl J Med 346(14):1061-1066. https://doi.org/10.1056/NEJMsa012528

Publisher's note Springer Nature remains neutral with regard to jurisdictional claims in published maps and institutional affiliations. 